\title{
PROJECTIVE ORDINAL SUMS OF LATTICES AND ISOTONE SECTIONS
}

\author{
RALPH FREESE AND J. B. NATION
}

\begin{abstract}
This note gives a complete characterization of when the ordinal sum of two lattices (the lattice obtained by placing the second lattice on top of the first) is projective. This characterization applies not only to the class of all lattices, but to any variety of lattices, and in particular, to the class of distributive lattices.

Lattices $\mathbf{L}$ with the property that every epimorphism onto $\mathbf{L}$ has an isotone section are also characterized.
\end{abstract}

In this paper we will determine when an ordinal sum of two lattices is projective in an arbitrary variety. The definition of projectivity most useful for the paper is the following one: A lattice $\mathbf{L}$ is projective in a variety $\mathcal{V}$ of lattices if $\mathbf{L} \in \mathcal{V}$ and whenever $\mathbf{K} \in \mathcal{V}$ and $f: \mathbf{K} \rightarrow \mathbf{L}$ is an epimorphism, there is a homomorphism $g: \mathbf{L} \rightarrow \mathbf{K}$ such that $f(g(a))=a$ for all $a \in L$. Note $g$ is one-to-one and $\rho=g f: \mathbf{K} \rightarrow \mathbf{K}$ is a retraction of $\mathbf{K}$; that is, $\rho$ is an endomorphism of $\mathbf{K}$ and $\rho^{2}=\rho$. The sublattice $\rho(\mathbf{K})=g(\mathbf{L})$ of $\mathbf{K}$ is isomorphic to $\mathbf{L}$. The image $g(\mathbf{L})$ is a retract of $\mathbf{K}$. In a slight abuse of terminology, we will also say $\mathbf{L}$ is a retract of $\mathbf{K}$. A lattice is projective in $\mathcal{V}$ if and only if it a retract of a free lattice $\mathbf{F}_{\mathcal{V}}(X)$ in $\mathcal{V}$. These remarks are well known.

Projective lattices (in the variety of all lattices) were characterized in [5, Theorem 5.7] by a conjunction of four conditions; see also [4]. This extended the results of A. Kostinsky [7], B. Jónsson [6], and R. McKenzie [8] who had characterized finitely generated projective lattices.

The map $g$ in the definition of projective lattice is an isomorphism and hence an order-preserving transversal to $\operatorname{ker} f$, also known as an isotone section. So the question arises which lattices $\mathbf{L}$ have the property that every epimorphism $\mathbf{K} \rightarrow \mathbf{L}$ has an isotone section? We will show that a lattice $\mathbf{L}$ has this property if and only if it satisfies the following condition.

For each $a \in L$ there are two finite sets $\mathrm{A}(a) \subseteq\{x \in L$ : $x \geq a\}$ and $\mathrm{B}(a) \subseteq\{x \in L: x \leq a\}$ such that if $a \leq b$ then $\mathrm{A}(a) \cap \mathrm{B}(b) \neq \emptyset$.

(A for 'above'; B for 'below.') A lattice, or more generally a partially ordered set, satisfying this condition is called finitely separable.

Date: May 29, 2014.

1991 Mathematics Subject Classification. 06B25, 06B05. 
It is easy to see that every countable lattice is finitely separable. This was used in [4] to show the following surprising result: the ordinal sum of two free lattices is projective if and only if one of them is finitely generated or both are countable.

In this note we give a complete characterization of when the ordinal sum of two lattices (the lattice obtained by placing the second lattice on top of the first) is projective. This characterization applies not only to the class of all lattices, but to any variety of lattices, and in particular to the class of distributive lattices.

Our main result is Theorem 12: An ordinal sum $\mathbf{L}=\mathbf{L}_{0} \dot{+} \mathbf{L}_{1}$ is projective in a variety $\mathcal{V}$ if and only if both $\mathbf{L}_{0}$ and $\mathbf{L}_{1}$ are projective and one of the following holds:

(1) $\mathbf{L}_{0}$ has a greatest element.

(2) $\mathbf{L}_{1}$ has a least element.

(3) $\mathbf{L}_{0}$ has a countable cofinal chain and $\mathbf{L}_{1}$ has a countable coinitial chain.

Recall than every chain in a projective lattice in any variety is countable, so the countability in the third condition is redundant here, but not for some of the lemmas.

The authors would like to give special thanks to George Bergman for his detailed comments and suggestions which greatly improved this paper.

\section{ISOTONE SECTIONS}

In this section we prove the theorem alluded to above. Much of this result is contained in Lemma 5.6 of [4].

Theorem 1. Let $\mathbf{L}$ be a lattice. The following are equivalent.

(1) L is finitely separable.

(2) Every epimorphism $\mathbf{K} \rightarrow \mathbf{L}$ has an isotone section.

(3) There is an epimorphism $f: \mathbf{F L}(X) \rightarrow \mathbf{L}$ which has an isotone section.

Proof. Since every lattice is an epimorphic image of a free lattice, (2) clearly implies (3). To see (3) implies (1) we use that, by Lemma 5.5 of 4], $\mathbf{F L}(X)$ is finitely separable with respect to a pair of mappings $A, B$. Let $g: \mathbf{L} \rightarrow$ $\mathbf{F L}(X)$ be an isotone section which exists by our assumption. For $a \in L$ set $A^{\prime}(a)=f(A(g(a)))$ and $B^{\prime}(a)=f(B(g(a)))$. If $a \leq b$ in $\mathbf{L}$, then $g(a) \leq g(b)$ in $\mathbf{F L}(X)$ and hence there exists an element $c \in A(g(a)) \cap B(g(b))$; the element $f(c)$ belongs to $A^{\prime}(a) \cap B^{\prime}(b)$. Thus $A^{\prime}$ and $B^{\prime}$ witness that $\mathbf{L}$ is finitely separable.

To see that (1) implies (2) let $\mathbf{L}$ be finitely separable with respect to a pair of mappings $A, B$. We can define a transfinite sequence $\left(M_{\gamma}: \gamma<\delta\right)$ of pairwise disjoint, nonempty and at most countable subsets of $L$ in the following way. Suppose that the sets $M_{\gamma}$ are already defined for all $\gamma$ less than an ordinal number $\gamma_{0}$ and put $M=\bigcup_{\gamma<\gamma_{0}} M_{\gamma}$. If $M=L$, put $\delta=\gamma_{0}$ 
and stop. If $M \subset L$, choose an element $a \in L-M$ arbitrarily and define $M_{\gamma_{0}}$ to be the least subset of $L$ containing $a$, disjoint from $M$, and such that $A(b) \cup B(b) \subseteq M \cup M_{\gamma_{0}}$ for any $b \in M_{\gamma_{0}}$.

The sets $M_{\gamma}$ define a partition of $L$. We shall write $a \sim b$ if $\{a, b\} \subseteq M_{\gamma}$ for some $\gamma$. There exists a well ordering $\sqsubseteq$ of $L$ such that $a \in M_{\gamma_{1}}, b \in M_{\gamma_{2}}$ and $\gamma_{1}<\gamma_{2}$ imply $a \sqsubseteq b$ and each $M_{\gamma}$ is a chain of type $\leq \omega$ with respect to the restriction of $\sqsubseteq$. This well ordering has the special property that if $a, b \in L$ and $b \in A(a) \cup B(a)$, then either $b \sqsubseteq a$ or else $a \sim b$ and $b$ comes only finitely many places after $a$.

Now suppose $f: \mathbf{K} \rightarrow \mathbf{L}$ is an epimorphism from a lattice $\mathbf{K}$ onto $\mathbf{L}$. For each $a \in L$ let $x_{a}$ be an element in $K$ such that $f\left(x_{a}\right)=a$. For $a \in L$ define $g(a) \in K$ by transfinite induction with respect to $\sqsubseteq$ as follows:

$$
\begin{aligned}
g(a)= & \left(x_{a} \wedge \bigwedge\{g(b): b \sim a, b \sqsubseteq a, a<b\} \wedge \bigwedge\{g(b): b \sqsubset a, b \in A(a)\}\right) \\
& \vee \bigvee\{g(b): b \sim a, b \sqsubseteq a, b<a\} \vee \bigvee\{g(b): b \sqsubset a, b \in B(a)\} .
\end{aligned}
$$

(Empty joins or meets are simply omitted. By construction, there are only finitely many elements with $b \sim a$ and $b \sqsubseteq a$; hence all the meets and joins are finite.) A straightforward induction shows that $f g(a)=a$ for any $a$.

Let us prove that $a \leq b$ implies $g(a) \leq g(b)$. We shall proceed by transfinite induction on the larger of the elements $a, b$ with respect to $\sqsubseteq$. There is an element $c \in A(a) \cap B(b)$.

First note that if $a \sqsubseteq c$ and $b \sqsubseteq c$, then, since $c \in A(a) \cap B(b)$, the special property of $\sqsubseteq$ gives $a \sim c \sim b$.

Now consider the case when $a \sqsubseteq b$. If $b \sqsubseteq c$, then by the above remarks $a \sim c \sim b$. This implies $g(a)$ is a joinand of $g(b)$, and so $g(a) \leq g(b)$. Hence we may assume $c \sqsubset b$, and thus by induction $g(a) \leq g(c)$. Also $g(c)$ is a joinand of $g(b)$ and so $g(c) \leq g(b)$ and hence $g(a) \leq g(b)$.

Next consider the case when $b \sqsubseteq a$ and either $a \sim b$ or $b \in A(a)$. Then $g(b)$ is a meetand of the first joinand of $g(a)$, so that the first joinand is $\leq g(b)$. Any other joinand of $g(a)$ is of the form $g(u)$ with $u \leq a \leq b$, so by induction $g(u) \leq g(b)$. Consequently, $g(a) \leq g(b)$.

Finally, let $b \sqsubseteq a$ and neither $a \sim b$ nor $b \in A(a)$. As we noted above, we cannot have $a \sqsubseteq c$ and $b \sqsubseteq c$ and this implies $c \sqsubset a$. So we have $g(a) \leq g(c)$ by the previous case and $g(c) \leq g(b)$ by induction.

\section{AN INTERPOLATION RESULT FOR RELATIVELY FREE LATTICES}

Theorem 1 essentially proves that every projective lattice is finitely separable. But we wish to show that every projective lattice in a variety $\mathcal{V}$ is finitely separable. We do this in this section. Along the way we prove an interpolation result of independent interest. Let $\mathcal{V}$ be a variety of lattices and let $\mathbf{F}_{\mathcal{V}}(X)$ denote the free $\mathcal{V}$-lattice generated by $X$. The following lemma is elementary. 
Lemma 2. Let $Z$ be a finite set and let $Y$ be a subset. Then every element of $\mathbf{F}_{\mathcal{V}}(Z)$ is either below $\bigvee Y$ or above $\bigwedge(Z-Y)$.

Proof. Let $\mathbf{S}=\left\{w \in \mathbf{F}_{\mathcal{V}}(Z): w \leq \bigvee Y\right.$ or $\left.w \geq \bigwedge(Z-Y)\right\}$. Then $\mathbf{S}$ is a sublattice of $\mathbf{F}_{\mathcal{V}}(Z)$ that contains all the generators, whence $\mathbf{S}=\mathbf{F}_{\mathcal{V}}(Z)$. Moreover, as long as the variety $\mathcal{V}$ is nontrivial, we can see that the disjunction is exclusive, i.e., $\bigwedge(Z-Y) \not \bigvee \bigvee Y$, by examining the homomorphism from $\mathbf{F}_{\mathcal{V}}(Z)$ to $\mathbf{2}$ which maps the elements of $Y$ to 0 and the other generators to 1 .

For $w \in \mathbf{F}_{\mathcal{V}}(X)$ define the rank of $w$, denoted $r(w)$, to be the the least integer $k$ such that there is a term of length $k$ representing $w$.

Lemma 3. Let $w \in \mathbf{F}_{\mathcal{V}}(X)$. If $w$ is in the sublattice generated by $U$ for some finite subset $U$ of $X$ and $w=w_{1} \vee \cdots \vee w_{n}$ is a minimal-rank expression for $w$, then each $w_{i}$ is in the sublattice generated by $U$.

Proof. To see this, suppose $t_{1}$ is a minimal length term representing $w_{1}$ and $y$ is a variable of $t_{1}$ which is not in $U$. Suppose one occurrence of $y$ is in a meet expression (the parent of this occurrence of $y$ in the term tree is a meet). Let $v=\bigvee Z$ where $Z$ is the set of all variables occurring in $t_{1}$. Let $w_{1}^{\prime}$ be $w_{1}$ with this single occurrence of $y$ replaced by $v$. Let $\sigma$ be the endomorphism of $\mathbf{F}_{\mathcal{V}}(X)$ induced by sending $y \mapsto v$ and $x \mapsto x$ otherwise. Clearly $\sigma$ is the identity map on the sublattice generated by $U$, and so $\sigma(w)=w$. Also $w_{1} \leq w_{1}^{\prime} \leq \sigma\left(w_{1}\right)$. Hence

$$
w \leq w_{1}^{\prime} \vee w_{2} \vee \cdots \vee w_{n} \leq \sigma\left(w_{1}\right) \vee \sigma\left(w_{2}\right) \vee \cdots \vee \sigma\left(w_{n}\right)=\sigma(w)=w .
$$

Therefore $w=w_{1}^{\prime} \vee w_{2} \vee \cdots \vee w_{n}$. But since $v$ is above all the other meetands in the meet expression containing it, we can eliminate $v$ from $w_{1}^{\prime}$. The resulting term is the same as $w_{1}$ with $y$ eliminated from this same place. This shows that there is a shorter expression for $w$, a contradiction.

For $Y$ a finite subset of $X$, let $\tau_{Y}$ be the endomorphism of $\mathbf{F}_{\mathcal{V}}(X)$ extending the map on $X$ :

$$
\tau_{Y}(z)= \begin{cases}z & \text { if } z \in Y \\ \bigvee Y & \text { if } z \notin Y\end{cases}
$$

Lemma 4. Let $\mathcal{V}$ be a nontrivial variety of lattices, let $Y$ be a finite subset of $X$, and let $\tau=\tau_{Y}$. Suppose $w \leq \bigvee Y$ in $\mathbf{F}_{\mathcal{V}}(X)$ and that $w$ is in the sublattice generated by $U$ for some subset $U$ of $X$. Then $w \leq \tau(w)$ and $r(\tau(w)) \leq r(w)$ and $\tau(w)$ is in the lattice generated by $Y \cap U$.

Proof. We induct on the rank of $w$. If $w$ is in $X$, then $w \leq \bigvee Y$ implies $w \in Y$ since $\mathcal{V}$ is nontrivial. Also $w \in U$ so the result holds in this case.

If $w=w_{1} \vee \cdots \vee w_{n}$, where this is a minimal-rank expression, then an easy inductive argument gives the desired result.

Let $Z$ be a finite subset of $X$ containing $Y$ such that $w$ lies in the sublattice generated by $Z$ and assume $w=w_{1} \wedge \cdots \wedge w_{n}$ is a minimal-rank expression. 
Since $\bigvee Y$ is meet prime by Lemma 2, there is an $i$ with $w_{i} \leq \bigvee Y$. By renumbering we may assume that $w_{i} \leq \bigvee Y$ for $1 \leq i \leq m$ and, by Lemma 2 , $w_{j} \geq \bigwedge(Z-Y)$ for $j>m$. By induction, $w_{i} \leq \tau\left(w_{i}\right)$ and $r\left(\tau\left(w_{i}\right)\right) \leq r\left(w_{i}\right)$ and $\tau\left(w_{i}\right)$ is in the sublattice generated by $U$, for $i \leq m$. For $j>m$, $\tau\left(w_{j}\right) \geq \tau(\bigwedge(Z-Y))=\bigvee Y$. Clearly the image of $\tau$ lies below $\bigvee Y$; hence $\tau\left(w_{i}\right) \leq \tau\left(w_{j}\right)$ if $i \leq m$ and $j>m$. Thus

$$
\tau(w)=\bigwedge_{i=1}^{n} \tau\left(w_{i}\right)=\bigwedge_{i=1}^{m} \tau\left(w_{i}\right) \geq \bigwedge_{i=1}^{m} w_{i} \geq w
$$

and $r(\tau(w)) \leq r(w)$. Since $\tau(w)=\bigwedge_{i=1}^{m} \tau\left(w_{i}\right)$ we have that $\tau(w)$ is in the sublattice generated by $U$.

Now suppose that $w \leq u$ in $\mathbf{F}_{\mathcal{V}}(X)$. Let $Y$ and $Z$ be a finite subsets of $X$ such that the lattice generated by $Y$ contains $u$ and the lattice generated by $Z$ contains $w$. Let $\tau=\tau_{Y}$. Since $u \leq \bigvee Y$, Lemma 4 gives

$$
w \leq \tau(w) \leq \tau(u)=u
$$

and $\tau(w)$ lies in the sublattice generated by $Y \cap Z$. In the case $w=u$, we see that if $w$ lies in the sublattice generated by $Y$ and by $Z$, then it lies in the sublattice generated by $Y \cap Z$. Thus, for each $w \in \mathbf{F}_{\mathcal{V}}(X)$ there is a unique smallest subset $Y$ of $X$ such that $w$ is in the sublattice generated by $Y$. We let $\operatorname{var}(w)$ denote this subset.

Now for our interpolation theorem.

Theorem 5. If $w \leq u$ in $\mathbf{F}_{\mathcal{V}}(X)$, there is a $v$ with $w \leq v \leq u$ such that $\operatorname{var}(v) \subseteq \operatorname{var}(w) \cap \operatorname{var}(u)$ and $\mathrm{r}(v) \leq \min (\mathrm{r}(w), \mathrm{r}(u))$.

Proof. If $\mathrm{r}(w) \leq \mathrm{r}(u)$, let $v=\tau_{\operatorname{var}(u)}(w)$ and the result follows from (1) and Lemma 4. If $\mathrm{r}(w)>\mathrm{r}(u)$, then a dual argument implies the result.

Using this interpolation theorem we can show that every lattice projective in $\mathcal{V}$ is finitely separable.

Theorem 6. If $\mathbf{L}$ is a projective lattice in $\mathcal{V}$ then $\mathbf{L}$ is finitely separable.

Proof. Finite separability is clearly preserved by retractions; thus it suffices to prove this theorem for $\mathbf{L}=\mathbf{F}_{\mathcal{V}}(X)$. For $a \in L$, let

$$
\mathrm{A}(a)=\left\{w \in \mathbf{F}_{\mathcal{V}}(X): w \geq a, \operatorname{var}(w) \subseteq \operatorname{var}(a), \text { and } \mathrm{r}(w) \leq \mathrm{r}(a)\right\}
$$

and define $\mathrm{B}(a)$ dually. The theorem follows from Theorem 5 .

\section{Projective ordinal Sums}

In this section we characterize when the ordinal sum of two lattices is projective in $\mathcal{V}$. We begin with some results of independent interest.

Lemma 7. Suppose $\mathbf{L}$ is finitely separable and that it is generated by a set $P$ of elements that are meet and join prime. Then $\mathbf{P}$ is finitely separable, where $\mathbf{P}$ is the partially ordered set on $P$ with the order inherited from $\mathbf{L}$. 
Proof. For each $a \in L$ let $t_{a}$ be a term with variables from $P$ of minimal length which evaluates to $a$. Suppose $t_{a}=t_{1} \vee \cdots \vee t_{n}$ and let $a_{i}$ be the interpretation of $t_{i}$ in $\mathbf{L}$. Inductively define $\operatorname{var}(a)$ to be the variables in $t_{a}$ together with $\operatorname{var}\left(a_{i}\right), i=1, \ldots, n$. (If $a \in P$, $\operatorname{var}(a)=\{a\}$. The case $t_{a}=t_{1} \wedge \cdots \wedge t_{n}$ is defined by duality.)

Let $A$ and $B$ be the functions that witness the finite separability of $\mathbf{L}$. For $p \in P$ define

$$
A^{\prime}(p)=\{r \in P: r \geq p \text { and } r \in \operatorname{var}(a) \text { for some } a \in A(p)\}
$$

Define $B^{\prime}(p)$ dually.

Suppose $p \leq q$ in $\mathbf{P}$. Then there is an $a \in L$ with $a \in A(p) \cap B(q)$. We show by induction on the complexity of $a$ that whenever $p \leq a \leq q$, there is an $r \in \operatorname{var}(a)$ with $p \leq r \leq q$. If $a \in P$ choose $r=a$. If $a=a_{1} \vee \cdots \vee a_{n}$ then since $p$ is join prime, $p \leq a_{i}$ for some $i$. Of course $a_{i} \leq q$ and $\operatorname{var}\left(a_{i}\right) \subseteq \operatorname{var}(a)$, so we can apply induction to obtain an $r \in \operatorname{var}(a)$ with $p \leq r \leq q$. Since the original element $a$ was in $A(p) \cap B(q)$, we have $r \in A^{\prime}(p) \cap B^{\prime}(q)$. A similar argument covers the case when $a$ is a meet.

Theorem 8. If $\mathbf{P}$ is a partially ordered set, then $\mathbf{F} v(\mathbf{P})$ (the $\mathcal{V}$ lattice freely generated by $P$ subject to the order relations of $\mathbf{P})$ is projective in $\mathcal{V}$ if and only if $\mathbf{P}$ is finitely separable.

Proof. Our proof follows the proof of Lemma 7 in [5].

Suppose $\mathbf{P}$ is finitely separable and $f: \mathbf{F}_{\mathcal{V}}(X) \rightarrow \mathbf{F}_{\mathcal{V}}(\mathbf{P})$ is an epimorphism. We can define an isotone map $g: \mathbf{P} \rightarrow \mathbf{F}_{\mathcal{V}}(X)$ such that $f g(p)=p$ essentially in the same way $g$ was defined in Theorem 11. By definition of $\mathbf{F}_{\mathcal{V}}(\mathbf{P}), g$ can be extended to homomorphism $\mathbf{F}_{\mathcal{V}}(\mathbf{P}) \rightarrow \mathbf{F}_{\mathcal{V}}(X)$ which of course is a retraction. This shows that $\mathbf{F}_{\mathcal{V}}(\mathbf{P})$ is projective in $\mathcal{V}$.

Now suppose $\mathbf{F}_{\mathcal{V}}(\mathbf{P})$ is projective in $\mathcal{V}$. By Theorem $6, \mathbf{F}_{\mathcal{V}}(\mathbf{P})$ is finitely separable. The elements of $P$ are join and meet prime in $\mathbf{F}_{\mathcal{V}}(\mathbf{P})$. (This can be seen by examining homomorphisms from $\mathbf{F}_{\mathcal{V}}(\mathbf{P})$ onto the two element lattice.) Hence by Lemma 7, $\mathbf{P}$ is finitely separable.

Lemma 9. An ordinal sum $\mathbf{L}_{0}+\mathbf{L}_{1}$ is finitely separable if and only if $\mathbf{L}_{0}$ and $\mathbf{L}_{1}$ are finitely separable and one of the following holds:

(1) $\mathbf{L}_{0}$ has a greatest element.

(2) $\mathbf{L}_{1}$ has a least element.

(3) $\mathbf{L}_{0}$ has a countable cofinal chain and $\mathbf{L}_{1}$ has a countable coinitial chain.

Proof. Assume that $\mathbf{L}_{0}$ and $\mathbf{L}_{1}$ are finitely separable and that at least one of the conditions (1), (2), or (3) holds. By hypothesis there are functions $\mathrm{A}_{\mathbf{L}_{i}}$ and $\mathrm{B}_{\mathbf{L}_{i}}$ that witness the finite separability of $\mathbf{L}_{i}, i=0$ and 1 . If $\mathbf{L}_{0}$ has a greatest element, say $m$, then for $a \in L_{0}$ let

$$
\mathrm{A}_{\mathbf{L}}(a)=\mathrm{A}_{\mathbf{L}_{0}}(a) \cup\{m\} \quad \text { and } \quad \mathrm{B}_{\mathbf{L}}(a)=\mathrm{B}_{\mathbf{L}_{0}}(a)
$$


and for $b \in L_{1}$ let

$$
\mathrm{A}_{\mathbf{L}}(b)=\mathrm{A}_{\mathbf{L}_{0}}(b) \quad \text { and } \quad \mathrm{B}_{\mathbf{L}}(b)=\mathrm{B}_{\mathbf{L}_{1}}(b) \cup\{m\} .
$$

Clearly the functions $A_{\mathbf{L}}$ and $B_{\mathbf{L}}$ have the desired properties. The same construction works if $m$ is the least element of $\mathbf{L}_{1}$.

So assume that (3) holds. Let $c_{0}<c_{1}<c_{2}<\cdots$ be a cofinal chain in $\mathbf{L}_{0}$ and let $d_{0}>d_{1}>d_{2}>\cdots$ be a coinitial chain in $\mathbf{L}_{1}$. Define

$$
\mathrm{A}^{\prime}\left(c_{i}\right)=\left\{d_{0}, d_{1}, \ldots, d_{i}\right\} \cup\left\{c_{i}\right\} \quad \text { and } \quad \mathrm{B}^{\prime}\left(d_{i}\right)=\left\{c_{0}, c_{1}, \ldots, c_{i}\right\} \cup\left\{d_{i}\right\} .
$$

Let $a \in L_{0}$ and suppose that $a \leq c_{i}$ but $a \not \leq c_{j}$ if $j<i$. Set $\mathrm{A}^{\prime}(a)=\mathrm{A}^{\prime}\left(c_{i}\right)$ and define

$$
\mathrm{A}_{\mathbf{L}}(a)=\mathrm{A}_{\mathbf{L}_{0}}(a) \cup \mathrm{A}^{\prime}(a)
$$

We define $\mathrm{B}_{\mathbf{L}}(b)$, for $b \in \mathbf{L}_{1}$, by dual considerations. Of course, $\mathrm{B}_{\mathbf{L}}(a)=$ $\mathrm{B}_{\mathbf{L}_{0}}(a)$ and $\mathrm{A}_{\mathbf{L}}(b)=\mathrm{A}_{\mathbf{L}_{0}}(b)$. With these definitions, it is easy to verify that $\mathbf{L}$ is finitely separable.

Conversely, assume that $\mathbf{L}=\mathbf{L}_{0} \dot{+} \mathbf{L}_{1}$ is finitely separable, with functions $\mathrm{A}$ and $\mathrm{B}$ witnessing its finite separability. For $x \in L_{i}$, let $\mathrm{A}_{\mathbf{L}_{i}}(x)=\mathrm{A}(x) \cap L_{i}$ and $\mathrm{B}_{\mathbf{L}_{i}}(x)=\mathrm{B}(x) \cap L_{i}$. It is easy to check that these functions give the finite separability of $\mathbf{L}_{0}$ and $\mathbf{L}_{1}$.

Suppose that conditions (1), (2), and (3) all fail. Since (3) fails, we may assume by duality that $\mathbf{L}_{0}$ has no countable cofinal chain. Inductively we construct a sequence $d_{0}>d_{1}>d_{2}>\cdots$ in $\mathbf{L}_{1}$ such that

$$
d_{n} \notin \operatorname{OrdFil}\left[\mathrm{B}_{\mathbf{L}_{1}}\left(d_{m}\right)\right] \quad \text { if } n>m
$$

where OrdFil $[S]$ denotes the order filter generated by a set $S$. (Dually, OrdIdl $[S]$ will denote the order ideal generated by $S$.) That this can be done follows from the fact that, for any $y \in L_{1}, L_{1}-\operatorname{OrdFil~} \mathrm{B}_{\mathbf{L}_{1}}(y)$ must be infinite since otherwise the meet of these elements and of $B_{\mathbf{L}_{1}}(y)$ would be a least element of $\mathbf{L}_{1}$, contrary to our assumption that condition (2) fails. Equation (2) immediately implies that

$$
\mathrm{B}_{\mathbf{L}_{1}}\left(d_{i}\right) \cap \mathrm{B}_{\mathbf{L}_{1}}\left(d_{j}\right)=\emptyset \quad \text { if } i \neq j .
$$

If OrdIdl $\left[\bigcup_{i=0}^{\infty} \mathrm{B}_{\mathbf{L}_{0}}\left(d_{i}\right)\right]=L_{0}$, then $\mathbf{L}_{0}$ would have a countable cofinal chain, contrary to our assumption. Thus let $c \notin \operatorname{OrdIdl}\left[\bigcup_{i=0}^{\infty} \mathrm{B}_{\mathbf{L}_{0}}\left(d_{i}\right)\right]$. Of course $c<d_{i}$ for every $i$, and hence there are elements $e_{i} \in L$ with

$$
e_{i} \in \mathrm{A}(c) \cap \mathrm{B}_{\mathbf{L}_{1}}\left(d_{i}\right) \text {. }
$$

By equation (3), these $e_{i}$ 's must be distinct. But this forces $\mathrm{A}(c)$ to be infinite.

The proof of Lemma 9 can be adapted to characterize when the ordinal sum of two finitely separable partially ordered sets is finitely separable. The proof is left to the reader.

Theorem 10. Let $\mathbf{P}_{0}$ and $\mathbf{P}_{1}$ be two finitely separable partially ordered sets. Then $\mathbf{P}_{0}+\mathbf{P}_{1}$ is finitely separable if and only if one of the following holds:

(1) $\mathbf{P}_{0}$ has a finite cofinal subset. 
(2) $\mathbf{P}_{1}$ has a finite coinitial subset.

(3) $\mathbf{P}_{0}$ has a countable cofinal subset and $\mathbf{P}_{1}$ has a countable coinitial subset.

Now let us turn our attention to projectivity in a nontrivial lattice variety $\mathcal{V}$.

Lemma 11. If the ordinal sum $\mathbf{L}=\mathbf{L}_{0} \dot{+} \mathbf{L}_{1}$ is projective in $\mathcal{V}$, then both $\mathbf{L}_{0}$ and $\mathbf{L}_{1}$ are projective in $\mathcal{V}$.

Proof. Let $f: \mathbf{M} \rightarrow \mathbf{L}_{0}$ be a surjective homomorphism. Let $\bar{f}: \mathbf{M} \dot{+} \mathbf{L}_{1} \rightarrow$ $\mathbf{L}_{0} \dot{+} \mathbf{L}_{1}$ agree with $f$ on $\mathbf{M}$ and the be the identity on $\mathbf{L}_{1}$. Since $\mathbf{L}_{0} \dot{+} \mathbf{L}_{1}$ is projective there is a homomorphism $\bar{g}: \mathbf{L}_{0} \dot{+} \mathbf{L}_{1} \rightarrow \mathbf{M} \dot{+} \mathbf{L}_{1}$ such that $\bar{f} \bar{g}$ is a retraction. Letting $g$ be the restriction of $\bar{g}$ to $\mathbf{L}_{0}$, it is clear that $f g$ is a retraction, proving $\mathbf{L}_{0}$ is projective. Similarly, $\mathbf{L}_{1}$ is projective.

Theorem 12. Let $\mathcal{V}$ be a nontrivial variety of lattices and let $\mathbf{L}=\mathbf{L}_{0} \dot{+} \mathbf{L}_{1}$, where $\mathbf{L}_{i} \in \mathcal{V}$ for $i=0,1$. Then $\mathbf{L}$ is projective in $\mathcal{V}$ if and only if both $\mathbf{L}_{0}$ and $\mathbf{L}_{1}$ are projective and one of the following holds:

(1) $\mathbf{L}_{0}$ has a greatest element.

(2) $\mathbf{L}_{1}$ has a least element.

(3) $\mathbf{L}_{0}$ has a countable cofinal chain and $\mathbf{L}_{1}$ has a countable coinitial chain.

Proof. Assume that $\mathbf{L}_{0}$ and $\mathbf{L}_{1}$ are projective in $V$ and that one of (1), (2), or (3) holds. Let $\mathbf{P}_{i}$ be $\mathbf{L}_{i}$ as a partially ordered set, and let $\mathbf{P}=\mathbf{P}_{0}+\mathbf{P}_{1}$. By Lemma 9, L is finitely separable, and so by Theorem 8, $\mathbf{F}_{\mathcal{V}}\left(\mathbf{P}_{0}\right) \dot{+} \mathbf{F}_{\mathcal{V}}\left(\mathbf{P}_{1}\right)$ is projective. Since $\mathbf{L}_{i}$ is projective, it is a retract of $\mathbf{F}_{\mathcal{V}}\left(\mathbf{P}_{i}\right)$, for $i=1,2$. Clearly these retractions can be patched together to show that $\mathbf{L}_{0} \dot{+} \mathbf{L}_{1}$ is a retract of $\mathbf{F}_{\mathcal{V}}\left(\mathbf{P}_{0}\right) \dot{+} \mathbf{F}_{\mathcal{V}}\left(\mathbf{P}_{1}\right)$. Thus $\mathbf{L}$ is projective in $\mathcal{V}$.

Conversely, suppose that $\mathbf{L}$ is projective in $\mathcal{V}$. By Lemma 11 , both $\mathbf{L}_{0}$ and $\mathbf{L}_{1}$ are projective in $\mathcal{V}$. Since $\mathbf{L}$ is projective in $\mathcal{V}$, by Theorem 6 it is finitely separable, whence by Lemma 9, at least one of the conditions (1), (2), or (3) holds.

As an application we show how Theorem 12 implies a theorem of Balbes [1] on projective distributive lattices.

Theorem 13. (Balbes [1]) Let $\mathbf{L}$ be a projective distributive lattice generated by $A \cup B$ where $A$ and $B$ are antichains, each element of $A$ is join irreducible, each element of $B$ is meet irreducible, and $a \leq b$ for each $a \in A$ and $b \in B$. Then either $A \cup B$ is countable or $A$ or $B$ is finite.

Proof. Let $\mathbf{L}_{0}$ be the sublattice generated by $A$ and $\mathbf{L}_{1}$ be the sublattice generated by $B$. Since every element of a projective distributive lattice is both a join of join irreducible elements and a meet of meet irreducible elements (see [2] or [5]), $L=L_{0} \cup L_{1}$ with $u \leq v$ for all $u \in L_{0}, v \in L_{1}$. If $L_{0} \cap L_{1} \neq \emptyset$, then $\mathbf{L}$ would not be projective, since the obvious map of 
$\mathbf{L}_{0} \dot{+} \mathbf{L}_{1}$ onto $\mathbf{L}$ has no section. Hence $L_{0} \cap L_{1}=\emptyset$ and $\mathbf{L}=\mathbf{L}_{0} \dot{+} \mathbf{L}_{1}$. By Theorem 12, one of the conditions (1), (2), or (3) must hold.

If $\mathbf{L}_{0}$ has a greatest element $u$ then, since $\mathbf{L}_{0}$ is generated by $A, u=\bigvee A^{\prime}$ for some finite subset $A^{\prime}$ of $A$. But in any distributive lattice join irreducible elements are join prime which implies $A=A^{\prime}$ and so is finite. Thus by duality we may assume that $\mathbf{L}_{0}$ has a countable cofinal chain and $\mathbf{L}_{1}$ has a countable coinitial chain. Since $\mathbf{L}_{0}$ is generated by $A$, each element $u_{i}$ of the cofinal chain lies in the sublattice generated by a finite subset $A_{i} \subseteq A$. Let $a \in A$. Then $a \leq u_{i}$ for some $u_{i}$ in the cofinal chain and so $a \leq u_{i} \leq \bigvee A_{i}$ Since $a$ is join prime and $A$ is an antichain, this implies that $a \in A_{i}$. Thus $A$ is the union of the $A_{i}$ 's and so is countable. Similarly, $B$ is countable.

The last theorem has a straightforward generalization to any variety $\mathcal{V}$ of lattices. We leave the proof to the reader.

Theorem 14. Let $\mathbf{L}$ be a projective lattice in $\mathcal{V}$ generated by $A \cup B$ where $A$ and $B$ are antichains, each element of $A$ is join prime, each element of $B$ is meet prime, and $a \leq b$ for each $a \in A$ and $b \in B$. Then either $A \cup B$ is countable or $A$ or $B$ is finite.

\section{Ordinal sums of more than two lattices}

The preceding results can be extended to the ordinal sum of a chain of lattices. Let $\mathbf{Q}$ be a totally ordered set, and let $\mathbf{L}_{q}(q \in Q)$ be lattices. The ordinal sum $\sum_{q \in Q} \mathbf{L}_{q}$ is the disjoint union $\dot{\bigcup}_{q \in Q} L_{q}$ endowed with the obvious order: if $x \in L_{q}$ and $y \in L_{r}$, then $x \leq y$ if $q<r$, or if $q=r$ and $x \leq y$ in $\mathbf{L}_{q}$. We remind the reader that projective lattices (indeed, finitely separable lattices) satisfy the countable chain condition (Theorem 5.69(4) of [4]), so for practical purposes we want to consider the case when $\mathbf{Q}$ is a countable chain.

Theorem 15. Let $\mathcal{V}$ be a nontrivial lattice variety, $\mathrm{Q}$ a totally ordered set, and $\mathbf{L}_{q}(q \in Q)$ lattices in $\mathcal{V}$. The ordinal sum $\mathbf{L}=\sum_{q \in Q} \mathbf{L}_{q}$ is projective in $\mathcal{V}$ if and only if

(1) each $\mathbf{L}_{q}$ is projective in $\mathcal{V}$, and

(2) $\sum_{q \in Q} \mathbf{L}_{q}$ is finitely separable.

Proof. That $\mathbf{L}$ projective implies (1) is a straightforward modification of Lemma 11. That $\mathbf{L}$ projective implies (2) is Theorem 6.

Conversely, let $\hat{\mathbf{L}}$ be $\mathbf{L}$ as an ordered set. By Theorem $8, \mathbf{F}_{\mathcal{V}}(\hat{\mathbf{L}})$ is projective in $\mathcal{V}$. Map $\mathbf{F}_{\mathcal{V}}(\hat{\mathbf{L}})$ onto $\mathbf{L}$ in the obvious way. As in the proof of Theorem 12 , each $\mathbf{L}_{q}$ is a retract of $\mathbf{F}_{\mathcal{V}}\left(\hat{\mathbf{L}_{q}}\right)$, and these maps can be patched together to get a retraction of $\mathbf{L}$ to $\mathbf{F}_{\mathcal{V}}(\hat{\mathbf{L}})$. Thus $\mathbf{L}$ is a retract of a projective lattice. Whence it is a retract of a free lattice in $\mathcal{V}$, and so projective.

We note one shortcoming of Theorem 15 as compared to the situation when $\mathbf{Q}=\mathbf{2}$. Lemma 9 characterizes when $\mathbf{L}_{0} \dot{+} \mathbf{L}_{1}$ is finitely separable, 
and this is readily extended to finite chains: an ordinal sum $\mathbf{L}_{0} \dot{+} \cdots \dot{+} \mathbf{L}_{n}$ is finitely separable if and only if each $\mathbf{L}_{i}$ is finitely separable and each consecutive pair $\mathbf{L}_{i}, \mathbf{L}_{i+1}$ satisfies at least one of the conditions (1), (2), or (3). But we do not have a good characterization of when $\sum_{q \in Q} \mathbf{L}_{q}$ is finitely separable for the case of $\mathbf{Q}$ being an infinite chain. This is a good open problem.

The next result provides plenty examples of finitely separable and projective lattices that are ordinal sums.

Theorem 16. Let $\mathbf{Q}$ be a countable chain. If $\mathbf{Q}$ has a least (resp. greatest) element, denote it by $z$ (resp. $t$ ).

If $\mathbf{L}_{q}(q \in Q)$ are finitely separable lattices, and each $\mathbf{L}_{q}$ has a least element $0_{\mathbf{L}_{q}}$ and a greatest element $1_{\mathbf{L}_{q}}$ (except possibly $\mathbf{L}_{z}$ has no least element and/or $\mathbf{L}_{t}$ has no greatest element), then the ordinal sum $\sum_{q \in Q} \mathbf{L}_{q}$ is finitely separable.

If in addition each $\mathbf{L}_{q}(q \in Q)$ is projective in a nontrivial lattice variety $\mathcal{V}$, then the ordinal sum $\sum_{q \in Q} \mathbf{L}_{q}$ is projective in $\mathcal{V}$.

Proof. By Theorem 15, it suffices to show that $\sum_{q \in Q} \mathbf{L}_{q}$ is finitely separable. Enumerate $Q=\left\{q_{i}: i \in \omega\right\}$. Let $A_{i}$ and $B_{i}$ denote the functions witnessing the finite separability of $\mathbf{L}_{q_{i}}$. For $x \in L_{q_{i}}$, let

$$
\begin{aligned}
& A(x)=A_{i}(x) \cup\left\{0_{\mathbf{L}_{j}}: j<i \text { and } q_{j}>q_{i}\right\} \cup\left\{1_{\mathbf{L}_{j}}: j \leq i \text { and } q_{j} \leq q_{i}\right\} \\
& B(x)=B_{i}(x) \cup\left\{1_{\mathbf{L}_{j}}: j<i \text { and } q_{j}<q_{i}\right\} \cup\left\{0_{\mathbf{L}_{j}}: j \leq i \text { and } q_{j} \geq q_{i}\right\}
\end{aligned}
$$

Now suppose $x \leq y$ with $x \in L_{i}$ and $y \in L_{j}$. This implies $q_{i} \leq q_{j}$. If $q_{i}=q_{j}$ then $A_{i}(x) \cap B_{i}(y)$ is nonempty. If $j<i$ then $0_{\mathbf{L}_{j}} \in A(x) \cap B(y)$, while if $j>i$ then $1_{\mathbf{L}_{i}} \in A(x) \cap B(y)$. Thus the ordinal sum is finitely separable.

On the other hand, observe that if $\mathbf{Q}$ is a countable chain and there is a covering pair $r \prec s$ such that $\mathbf{L}_{r}$ has no greatest element and no countable cofinal chain, while $\mathbf{L}_{s}$ has no least element and no countable coinitial chain, then $\sum_{q \in Q} \mathbf{L}_{q}$ is not finitely separable. For in this event we can let $\mathbf{K}_{0}=$ $\sum_{q \leq r} \mathbf{L}_{q}$ and $\mathbf{K}_{1}=\sum_{q \geq s} \mathbf{L}_{q}$, and then apply Lemma 9 to $\mathbf{K}_{0} \dot{+} \mathbf{K}_{1}$.

We conclude with a nice example. Let $\kappa$ be an uncountable cardinal. Then by Theorem 12 the lattice $\mathbf{F}_{\mathcal{V}}(\kappa) \dot{+} \mathbf{1} \dot{+} \mathbf{F}_{\mathcal{V}}(\kappa)$ is projective. But if we remove the middle element, then by the preceding observation $\mathbf{F}_{\mathcal{V}}(\kappa) \dot{+} \mathbf{F}_{\mathcal{V}}(\kappa)$ is not projective.

\section{REFERENCES}

[1] R. Balbes. Generating sets for catalytic and projective distributive lattices. Algebra Universalis, 30:262-268, 1993.

[2] R. Balbes and A. Horn. Projective distributive lattices. Pacific J. Math., 33:421-435, 1970.

[3] G. M. Bergman and G. Grätzer. Isotone maps on lattices. Algebra Universalis, 68(12):17-37, 2012. 
[4] Ralph Freese, Jaroslav Ježek, and J. B. Nation. Free Lattices. Amer. Math. Soc., Providence, 1995. Mathematical Surveys and Monographs, vol. 42.

[5] Ralph Freese and J. B. Nation. Projective lattices. Pacific J. Math., 75:93-106, 1978.

[6] B. Jónsson and J. B. Nation. A report on sublattices of a free lattice. In Contributions to universal algebra, pages 233-257. North-Holland Publishing Co., Amsterdam, 1977. Coll. Math. Soc. János Bolyai, vol. 17.

[7] A. Kostinsky. Projective lattices and bounded homomorphisms. Pacific J. Math., 40:111-119, 1972.

[8] R. McKenzie. Equational bases and non-modular lattice varieties. Trans. Amer. Math. Soc., 174:1-43, 1972.

UNIVERSiTy OF HAWAiI, HonOlulu, HI 96822

E-mail address: ralph@math.hawaii.edu

University OF HaWAit, HonOlulu, HI 96822

E-mail address: jb@math.hawaii.edu 